\title{
MOLECULAR DOCKING STRATEGY FOR MULTI-TARGET INHIBITOR DISCOVERY OF SELECTED PLANT CONSTITUENTS IN BAUHINIA ACUMINATA
}

\author{
BAUHINIA ACUMINATA'DAKİ SEÇILLMIŞ FITTO BILEŞENLERIN ÇOK HEDEFLI \\ INHIBITTÖR KEŞFI IÇINN MOLEKÜLER YERLEŞTIRME STRATEJISİ
}

\begin{abstract}
Muni Sireesha SUNKARA ${ }^{1} \mathbb{D}$, Vinutha KUCHANA ${ }^{1} \mathbb{D}$, Neelima KUDUMULA $^{1^{*}}$
${ }^{1}$ Osmania University, Sarojini Naidu Vanita Pharmacy Mahavidyalaya, Department of Pharmaceutical Chemistry, 500017, Hyderabad, India
\end{abstract}

\begin{abstract}
Objective: Traditional medicine is often considered to be a kind of complementary or alternative medicine (CAM) nowadays. Therefore, documenting and identifying the herbs that are effective in treating various diseases is vital for future disease control programs. This study aims to perform a molecular docking analysis of the thirteen plant components in Bauhinia acuminata against the target proteins in lung cancer (PDB IDs: $2 I T Y)$, breast cancer (1A52), diabetes (3LAU), obesity (ITO2), inflammation (5COX) and corona viral infections (6VYO).
\end{abstract}

Material and Method: All the plant components used for the present study were retrieved from the plant Bauhinia acuminata and were evaluated for their biological activity results using molinspiration. Further insilico docking analysis was performed using AutoDock Vina software and the binding interactions were visualized using Discovery studio program.

Result and Discussion: The docking scores and analysis of the interactions of the plant components with targets suggest that all the selected plant components showed excellent binding to the chosen targets when compared to that of the standard drugs. As a result of the docking process on 6 different targets, the selected plant components like Quercetin, Beta-sitosterol, and Rheagenine were observed to show good binding energy values against all the 5 targets except 6VYO as shown in (Table 9). These results can further pave the way for getting better insights in identifying and designing potential lead candidates.

Keywords: AutoDock Vina, Bauhinia acuminata, discovery studio, molecular docking, plant components

$\ddot{\mathbf{O Z Z}}$

\footnotetext{
* Corresponding Author/Sorumlu Yazar: Neelima Kudumula e-mail / e-posta: neelima_kudumula@ yahoo.com, Phone / Tel.: +919502792574
}

Submitted / Gönderilme: 25.08.2021

Accepted / Kabul: 16.12.2021 
Amaç: Günümüzde geleneksel tıp genellikle bir tür tamamlayıcl veya alternatif tıp (CAM) olarak kabul edilmektedir. Bu nedenle, çeşitli hastalıkların tedavisinde etkili olan bitkilerin belgelenmesi ve tanımlanması gelecekteki hastalık kontrol programları için hayati öneme sahiptir. Bu çalışma Bauhiniaacuminata'daki on üç bitki bileşeninin akciğer kanseri (PDB IDs: 2ITY), meme kanseri (1A52), diyabet (3L4U), obezite (ITO2), inflamasyon (5COX) ve korona viral enfeksiyonlarındaki (6VYO) hedef proteinlere karşı moleküler kenetlenme analizini gerçekleştirmeyi amaçlamaktadır.

Gereç ve Yöntem: Bu çalışmada kullanılan tüm bitki bileşenleri Bauhiniaacuminata bitkisinden alınmış ve molinspirasyon kullanılarak biyolojik aktivite sonuçları açısından değerlendirilmiştir. Ayrıca AutoDockVına yazılımı kullanılarak siliko içi yerleştirme analizi yapıldı ve bağlanma etkileşimleri Discoverystudio programı kullanılarak görselleştirildi.

Sonuç ve Tartışma: Yerleştirme puanları ve bitki bileşenlerinin hedeflerle etkileşimlerinin analizi, seçilen tüm bitki bileşsenlerinin standart ilaçlarınkine kiyasla seçilen hedeflere mükemmel bağlanma gösterdiğini göstermektedir. 6 Farkl hedefe kenetlenme işlemi sonucunda, Quercetin, Beta-sitosterol ve Rheagenin gibi seçilen bitki bileşenlerinin, gösterildiği gibi 6 VYO hariç tüm 5 hedefe karşı iyi bağlanma enerjisi değerleri gösterdiği gözlenmiştir (Tablo 9). Bu sonuçlar potansiyel potansiyel adayları belirleme ve tasarlama konusunda daha iyi kavrayışlar elde etmenin önünü daha da açabilir.

Anahtar Kelimeler: AutoDock Vina, Bauhinia acuminata, bitki bileşenleri, discovery studio, moleküler yerleştirme

\section{INTRODUCTION}

The World Health Organization (WHO) defines traditional medicine as: "the sum of total knowledge, practices, and skills based on the historical theories, beliefs, and experiences in indigenous to various cultures that are used to maintain the human or animal health and to prevent, diagnose, improve, or treat physical/mental illnesses" [1].

Herbal remedies are widely used in both developing and developed world countries to treat various illnesses indispensable [2]. The WHO reported, to treat their illnesses about $80 \%$ of the world's population are depending primarily on traditional medicines. Traditional medicine is often considered to be a kind of complementary or alternative medicine (CAM) [3] nowadays. Herbal medicines include herbal preparations, raw herbs, and finished herbal products, as well as additives derived from different kinds of plant parts/herbs. Many advantages are shown by the active components of these herbs, like lower toxicity and allergenicity than when compared to some commercial medications, regulating immunological responses, and causing viral destruction [4]. In the research trials [5], various common herbs have been used to prevent viral infections, and their efficacy has been demonstrated. Therefore, documenting and identifying the herbs that are effective in treating various contagious diseases is vital for future disease control programs.

Bauhinia acuminata is an ever green shrub belonging to the family of Fabaceae grown in the areas of South east Asia such as Malaysia, Indonesia, or the Philippines. For conventional drugs, bark, leaves, stem, blooms, and roots have been utilized. In India, it is a traditional plant, and its extract in studies have shown that Bauhinia acuminata have significant biological activities such as in the treatment of lung cancer [6], breast cancer [7], anti-diabetic [8], anti-obesity [9], anti-inflammatory [10]. Based on 
the reported anti-lung cancer, anti-breast, anti-diabetic, anti-obesity activities, molecular docking studies have been planned to establish the contribution of the activity by the plant components.

Bauhinia acuminata has been chemically studied and reported wherein the important chemical constituents isolated from Bauhinia acuminata which are depicted in (Table 1) are chosen for our studies. All the plant components will be evaluated in this study on the docking behavior of EGFR [11], ESTROGEN ALPHA RECEPTOR [12], ALPHA GLUCOSIDASE [13], HMG COA [14], 5COX [15], SAR COV-2 [16] using an In silico molecular docking analysis with AutoDock Vina software and also an investigation on the enzymes binding sites using Discovery Studio Version 3.5.

\section{MATERIAL AND METHOD}

The X-ray crystallographic structures of six different targets 2ITY (Lung cancer), 1A52 (Breast cancer), 3L4U (Diabetes), IT02 (Obesity), 5COX (Inflammation), 6VYO (Corona Virus infection) were retrieved from protein databank and saved as a Brookhaven protein data bank file [17]. For all the six targets, the standard ligands were selected from the literature review and downloaded from Pubchem Database as depicted in (Figure 1). The 2D structures of all thirteen plant components were sketched using ACD/Chemsketch Software as shown in (Table 1). The generated ligands were cleaned and performed 3D optimization and then saved in the MDL Molfile format. The ligands were then converted to a PDB file format using the Open Babel chemistry toolbox. AutoDock Vina [18] (Academic version.1.2.0, Molecular Graphics Lab (now CCSB) at The Scripps Research Institute) was used for molecular docking studies. A grid was generated around the co-crystallized ligand. The coordinates were generated with the help of MGL Tools and Pharmit: interactive exploration of chemical space [19]. Prepared pdbqt files for both target and ligands by adding Polar hydrogens and Gasteiger charges. Created in-house batch file of ligands and target and then docking was performed in the absence of water molecules for all the plant components [20]. The molecules were then analyzed after docking and visualized in the Discovery Studio Version 3.5 [21] for the interactions with the active site amino acids. Further to optimize the docking process all the crystal structures of the targets compared with the standard ligands. Ligand interactions were shown in (Figure 1 to Figure 6).

\section{RESULT AND DISCUSSION}

Bioactivity score is a computational approach that can be used to determine whether or not a particular molecule is similar to the known drugs in its structural features and molecular properties. According to the bioactivity score, if $>0$ is active; if $(-5.0$ to -0.0$)$ is moderately active, and if $<-5.0$ is inactive. In the present study, all the thirteen plant components of Bauhinia acuminata showed active scores $(>0)$ toward enzyme inhibitors descriptors. However, for other descriptors, these compounds 
exhibited active to moderate active scores with none showing inactive score $(<-5.0)$, as shown in (Table

2) which is already reported and is considered for our present work [22].

Table 1. Names and structures of the plant constituents of Bauhina acuminata

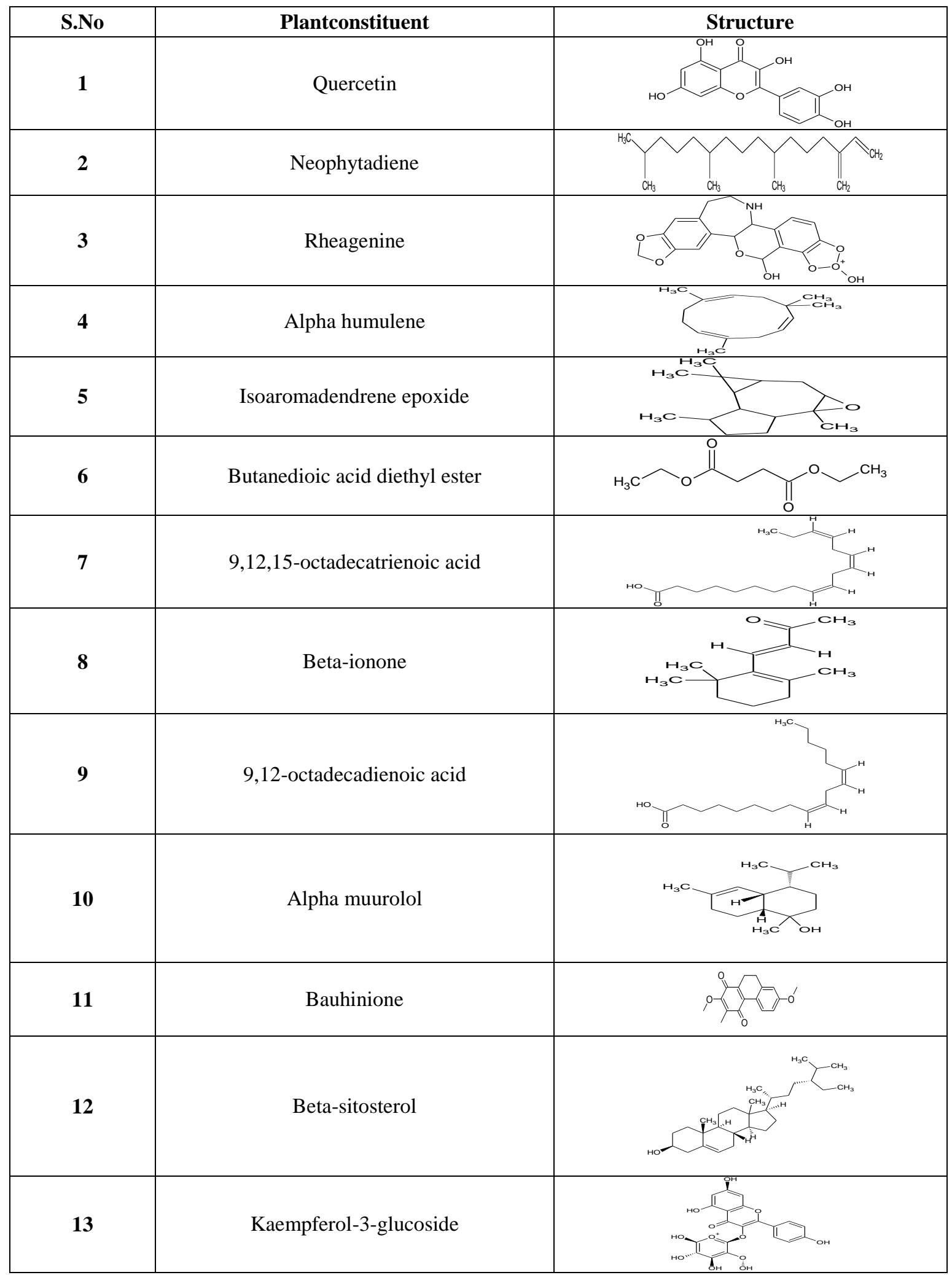


Table 2. Computed biological activity results of the plant constituents of Bauhinia acuminata by molinspiration

\begin{tabular}{|c|c|c|c|c|c|c|c|}
\hline S.No & $\begin{array}{c}\text { Plant } \\
\text { Constituent }\end{array}$ & $\begin{array}{c}\text { GPCR } \\
\text { Ligand }\end{array}$ & $\begin{array}{c}\text { Ion } \\
\text { Channel } \\
\text { Modulator }\end{array}$ & $\begin{array}{c}\text { Kinase } \\
\text { Inhibitor }\end{array}$ & $\begin{array}{c}\text { Nuclear } \\
\text { Receptor } \\
\text { Ligand }\end{array}$ & $\begin{array}{c}\text { Protease } \\
\text { Inhibitor }\end{array}$ & $\begin{array}{c}\text { Enzyme } \\
\text { Inhibitor }\end{array}$ \\
\hline $\mathbf{1}$ & Quercetin & -0.06 & -0.19 & 0.28 & 0.36 & -0.25 & 0.28 \\
\hline $\mathbf{2}$ & Neophytadiene & -0.12 & -0.02 & -0.35 & 0.2 & -0.11 & 0.14 \\
\hline $\mathbf{3}$ & Rheagenine & 0.21 & 0.13 & -0.14 & -0.09 & 0.02 & 0.17 \\
\hline $\mathbf{4}$ & Alpha humulene & -0.14 & 0.02 & -0.93 & 0.34 & -0.67 & 0.31 \\
\hline $\mathbf{5}$ & $\begin{array}{c}\text { Isoaromadendrenee } \\
\text { poxide }\end{array}$ & -0.39 & -0.37 & -0.69 & -0.01 & 0.02 & -0.05 \\
\hline $\mathbf{6}$ & $\begin{array}{c}\text { Butanedioicaciddiet } \\
\text { hyl ester }\end{array}$ & -0.93 & -0.35 & -1.19 & -0.91 & -0.92 & -0.46 \\
\hline $\mathbf{7}$ & $\begin{array}{c}9,12,15- \\
\text { octadecatrienoic } \\
\text { acid }\end{array}$ & 0.29 & 0.17 & -0.16 & 0.31 & 0.12 & 0.38 \\
\hline $\mathbf{8}$ & Beta-ionone & -0.9 & -0.26 & -1.34 & 0.25 & -0.79 & 0.28 \\
\hline $\mathbf{9}$ & $\begin{array}{c}9,12- \\
\text { octadecadienoic } \\
\text { acid }\end{array}$ & 0.29 & 0.17 & -0.16 & 0.31 & 0.12 & 0.38 \\
\hline $\mathbf{1 0}$ & Alpha muurolol & -0.09 & 0.05 & -0.87 & 0.39 & -0.63 & 0.4 \\
\hline $\mathbf{1 1}$ & Bauhinione & -0.13 & -0.18 & 0.1 & 0.06 & -0.29 & 0.34 \\
\hline $\mathbf{1 2}$ & Beta-sitosterol & 0.14 & 0.04 & -0.51 & 0.73 & 0.07 & 0.51 \\
\hline $\mathbf{1 3}$ & $\begin{array}{c}\text { Kaempferol-3- } \\
\text { glucoside }\end{array}$ & 0.06 & -0.05 & 0.1 & 0.2 & -0.05 & 0.41 \\
\hline
\end{tabular}

In this in silico study, we have seen that thirteen plant components of Bauhinia acuminata exhibited the most negative value of docking score toward 2ITY, 1A52, 3L4U, IT02, 5COX means the best affinity was formed except for 6VYO. The docking scores and analysis of the interactions of the plant components with targets suggest that all the selected plant components showed excellent binding to the chosen targets when compared to that of the standard drugs.

Molecular docking interactions of all plant components with the target 1A52 were elucidated, and it was observed that various hydrogen bond interactions with aminoacids LEUA:387, ILEA:424, META:421, HISA:524, GLYA:521, GLUA:353, ARGA:394, ARGA:434, LEUA:384, META:388, META:343, THRA:347, LEUA:349, LEUA:354, LEUA:428, META:388, TRPA:383, ARGA:515, GLUA:323, TRPA:393 were determined. Binding energies of all the compounds were found between 5.6 to $-8.6 \mathrm{kcal} / \mathrm{mol}$ which were illustrated in (Table 3). All the plant components except Butanedioic acid diethyl ester showed more binding energies than the standard Tamoxifen (-6.2 kcal/mol).

All the plant components when docked with target 2ITY the following hydrogen bond interactions with aminoacids LEUA:844, PROA:794, LYSA:745, META:793, LYSA:745, LEUA:718, VALA:726, ASPA:855, GLYA:719, LEUA:844, GLNA:791, GLUA:762, THRA:854, SERA:720, ASPA:855 were determined. Binding energies of all the compounds were found between -5.1 to $-9.2 \mathrm{kcal} / \mathrm{mol}$ which were illustrated in (Table 4). Among all compounds, Rheagenine, Beta-sitosterol, Bauhinione, Quercetin, Kaempferol-3-glucoside, and Isoaromadendrene epoxide showed more binding energies than the standard Erlotinib ( $-7.1 \mathrm{kcal} / \mathrm{mol})$. 
Table 3. Calculated binding energy values and interaction properties of the plant constituents of the phyto-constituents of Bauhinia acuminata the target 2ITY

\begin{tabular}{|c|c|c|c|c|}
\hline S.No & Plant constituent & $\begin{array}{l}\text { Binding } \\
\text { Energy } \\
(\mathbf{k c a l} / \mathbf{m o l})\end{array}$ & $\begin{array}{l}\text { Hydrogen Bond } \\
\text { Interactions }\end{array}$ & Alkyl/ $\pi$ Alkyl Interactions \\
\hline 1 & Quercetin & -8 & $\begin{array}{c}\text { META:793, } \\
\text { GLNA:791, ASPA:855, } \\
\text { GLUA:762 } \\
\end{array}$ & $\begin{array}{c}\text { LEUA:844, LEUA:718, ALAA:743, } \\
\text { LYSA:745, VALA:726 }\end{array}$ \\
\hline 2 & Neophytadiene & -5.3 & META:793 & $\begin{array}{c}\text { LEUA:844, LEUA:718, ALAA:743, } \\
\text { VALA:726 }\end{array}$ \\
\hline 3 & Rheagenine & -9.2 & LEUA:844, META:793 & ALAA:743, VALA:726 \\
\hline 4 & Alpha humulene & -6.7 & LEUA:718 & VALA:726 \\
\hline 5 & Isoaromadendreneepoxide & -7.4 & No Interactions & LEUA:858, LEUA:747, PHEA:723 \\
\hline 6 & $\begin{array}{l}\text { Butanedioicaciddiethyl } \\
\text { ester }\end{array}$ & -5.5 & $\begin{array}{l}\text { GLYA:719, SERA:720, } \\
\text { PHEA:723, THRA:725, } \\
\text { LYSA:745, ASPA:855 }\end{array}$ & LEUA:718, VALA:726, CYSA:797 \\
\hline 7 & $\begin{array}{l}\text { 9,12,15-octadecatrienoic } \\
\text { acid }\end{array}$ & -5.6 & ASPA: 855 & $\begin{array}{l}\text { VALA:726, LEUA:844, LEUA:718, } \\
\text { LEUA:792, ALAA:743, META:793 }\end{array}$ \\
\hline 8 & Beta-ionone & -6.4 & $\begin{array}{l}\text { VALA:726, LEUA:844, } \\
\text { META:793 }\end{array}$ & No Interactions \\
\hline 9 & 9,12-octadecadienoic acid & -5.1 & $\begin{array}{c}\text { LYSA: } 745, \text { ASPA: } 855, \\
\text { THRA: } 854 \\
\end{array}$ & $\begin{array}{c}\text { LEUA:844, LEUA:718, ALAA:743, } \\
\text { VALA:726 } \\
\end{array}$ \\
\hline 10 & Alpha muurolol & -6.8 & No Interactions & $\begin{array}{l}\text { LEUA:844, CYSA:775, LEUA:718, } \\
\text { LEUA:792, ALAA:743, VALA:726 }\end{array}$ \\
\hline 11 & Bauhinione & -8.1 & LYSA:745, ASPA:855 & $\begin{array}{c}\text { ALAA:743, LEUA:844, LEUA:718, } \\
\text { VALA:726 }\end{array}$ \\
\hline 12 & Beta-sitosterol & -8.4 & PROA:794, LEUA:844 & ALAA:743, LEUA:718, VALA:726 \\
\hline 13 & Kaempferol-3-glucoside & -7.9 & META:793, GLUA:762 & $\begin{array}{c}\text { LEUA:844, LEUA:718, ALAA:743, } \\
\text { VALA:726, LYSA:745 } \\
\end{array}$ \\
\hline 14 & $\begin{array}{l}\text { ERLOTINIB } \\
\text { (STANDARD) }\end{array}$ & -7.1 & $\begin{array}{l}\text { LYSA:745, THRA:854, } \\
\text { GLYA:721, GLYA:719 }\end{array}$ & $\begin{array}{c}\text { ALAA:743, LEUA:844, LEUA:718, } \\
\text { VALA:726 }\end{array}$ \\
\hline
\end{tabular}

All the plant components when docked with target 3L4U target the following hydrogen bond interactions with amino acids ASPA:203, THRA:204, LYSA:480, THRA:205, GLUA:333, TYRA:299, HISA:600, ASPA:203, THRA:205, GLNA:603, ASPA:203, GLUA:404, ASPA:542, ARGA:526, ARGA:542, THRA:205, LYSA:480, PHEA:450, GLUA:404, ASPA:443. Binding energies of all the compounds were found between -5.3 to $-8.3 \mathrm{kcal} / \mathrm{mol}$ which were illustrated in (Table 5). Among all compounds Rheagenine, Beta-sitosterol and Quercetin showed more binding energies than the standard Acarbose (-7.1 kcal/mol).

All the plant components made hydrogen bond interactions with aminoacids ASNB:375, GLYA:536, TYRB:373, ASNA:375, ASNB:375, PHEB:142, GLYB:225, GLNA:241, PROB:538, LYSA:333, GLYA:227, HISB:226, ASNA:375, GLNB:374, VALB:228, ARGB:376, TRPB:139, GLNA:241, ARGB:376, ARGA:376, GLYA:225, LYSA:333, GLUB:140, LEUB:145, ASNB:144, LEUA:145, TYRA:373 in the active site of 5COX target. Binding energies of all the compounds were found between -5 to $-9.9 \mathrm{kcal} / \mathrm{mol}$ which were illustrated in (Table 6). Among all compounds, Rheagenine, Beta-sitosterol, Bauhinione, Quercetin, Kaempferol-3-glucoside, Iso aromadendrene 
epoxide, alpha Muurolol, Alpha Humulene, Beta-Ionone, and Butanedioic acid diethyl ester showed more binding energies than the standard Aspirin $(-5.8 \mathrm{kcal} / \mathrm{mol})$.

Table 4. Calculated binding energy values and interaction properties of the plant constituents of Bauhinia acuminata the target 1A52

\begin{tabular}{|c|c|c|c|c|}
\hline S. No & Plant Constituent & $\begin{array}{c}\text { Binding } \\
\text { energy } \\
\text { (kcal/mol) }\end{array}$ & $\begin{array}{c}\text { Hydrogen } \\
\text { Bond Interactions }\end{array}$ & Alkyl/ $\pi$ Alkyl Interactions \\
\hline 1 & Quercetin & -8.5 & $\begin{array}{c}\text { GLYA:521, GLUA:353, } \\
\text { ARGA:394 }\end{array}$ & $\begin{array}{c}\text { META:421, ILEA:424, } \\
\text { LEUA:391, ALAA:350, } \\
\text { LEUA:387, PHEA:404, LEUA:384 }\end{array}$ \\
\hline 2 & Neophytadiene & -6.8 & $\begin{array}{l}\text { LEUA:428, META:388, } \\
\text { TRPA:383 }\end{array}$ & $\begin{array}{l}\text { LEUA:525, LEUA:387, } \\
\text { LEUA:391, PHEA:404, } \\
\text { ALAA:350, LEUA:384 }\end{array}$ \\
\hline 3 & Rheagenine & -7.2 & No Interactions & LEUA:354, TRPA:383, ASPA:351 \\
\hline 4 & Alpha humulene & -8 & LEUA:384, META:388 & No Interactions \\
\hline 5 & Isoaromadendreneepoxide & -8.3 & ARGA:434 & $\begin{array}{c}\text { LEUA:509, HISA:513, ILEA:510, } \\
\text { PHEA:404, LEUA:384 }\end{array}$ \\
\hline 6 & $\begin{array}{l}\text { Butanedioicaciddiethyl } \\
\text { ester }\end{array}$ & -5.6 & GLUA:323, TRPA:393 & $\begin{array}{c}\text { LEUA:403, ARGA:394, } \\
\text { META:396, PROA:324, ILEA:326 }\end{array}$ \\
\hline 7 & $\begin{array}{l}\text { 9,12,15-octadecatrienoic } \\
\text { acid }\end{array}$ & -7 & HISA:524, GLYA:521 & $\begin{array}{c}\text { LEUA:346, PHEA:404, } \\
\text { META:388, LEUA:391, } \\
\text { LEUA:387, ALAA:350, } \\
\text { LEUA:384, LEUA:525, TRPA:383 }\end{array}$ \\
\hline 8 & Beta-ionone & -7.2 & THRA:347 & $\begin{array}{c}\text { ALAA:350, LEUA:387, } \\
\text { PHEA:404, LEUA:391 }\end{array}$ \\
\hline 9 & 9,12-octadecadienoic acid & -6.8 & ARGA:394, LEUA:387 & $\begin{array}{l}\text { TRPA:383, ALAA:350, } \\
\text { LEUA:384, LEUA:525, } \\
\text { PHEA:404, ILEA:424, } \\
\text { META:421, LEUA:391, } \\
\text { META:388, LEUA:346 }\end{array}$ \\
\hline 10 & Alpha muurolol & -7.9 & $\begin{array}{l}\text { META:343, THRA:347, } \\
\text { LEUA:349 }\end{array}$ & $\begin{array}{l}\text { ALAA:350, LEUA:387, } \\
\text { PHEA:404, LEUA:525, } \\
\text { LEUA:384, TRPA:383, } \\
\text { LEUA:346, META:421 }\end{array}$ \\
\hline 11 & Bauhinione & -8.6 & $\begin{array}{l}\text { LEUA:387, ILEA:424, } \\
\text { META:421, HISA:524 }\end{array}$ & $\begin{array}{c}\text { LEUA:346, ALAA:350, } \\
\text { PHEA:404 }\end{array}$ \\
\hline 12 & Beta-sitosterol & -7 & LEUA:354 & $\begin{array}{l}\text { VALA:376, ILEA:358, } \\
\text { LEUA:372, TRPA:383 }\end{array}$ \\
\hline 13 & Kaempferol-3-glucoside & -8.4 & ARGA:394, GLUA:353 & $\begin{array}{l}\text { ILEA:424, META:421, } \\
\text { LEUA:387, ALAA:350, } \\
\text { LEUA:391 }\end{array}$ \\
\hline 14 & $\begin{array}{l}\text { TAMOXIFEN } \\
\text { (STANDARD) }\end{array}$ & -6.2 & ARGA:515 & LEUA:508,LEUA:479, ILEA:451 \\
\hline
\end{tabular}

Molecular docking interactions of the plant components were elucidated with 6VYO target, were various hydrogen bond interactions like ALAA:50, TYRA:111, ARGA:88, TYRA:109, ALAA:55, ARGA:107, ASNB:153, VALA:158, ILEB:157, ILEB:146, THRB:76, ASNB:77, ALAA:55, ARGA:107, ASNB:75, SERA:105, SERB:79, THRB:141, HISA:59, ASPB:81 were observed. Binding energies of all the compounds were found between -5.7 to $-9.7 \mathrm{kcal} / \mathrm{mol}$ which were illustrated in (Table 7). Among all compounds, Isoaromadendreneepoxide showed more binding energy than the standard N3 inhibitor of 6LU7 (-5.8 kcal/mol). 
Table 5. Calculated binding energy values and interaction properties of the plant constituents of Bauhinia acuminata the target 3L4U

\begin{tabular}{|c|c|c|c|c|}
\hline S.No & Plant Constituent & $\begin{array}{c}\text { Bindin } \\
\text { genergy } \\
(\mathrm{kcal} / \mathrm{mol})\end{array}$ & $\begin{array}{l}\text { Hydrogen Bond } \\
\text { Interactions }\end{array}$ & Alkyl/ $\pi$ Alkyl Interactions \\
\hline 1 & Quercetin & -7.3 & ASPA:542 & $\begin{array}{c}\text { ASPA:443, META:444, } \\
\text { PHEA:575, TYRA:299, } \\
\text { TRPA:406 }\end{array}$ \\
\hline 2 & Neophytadiene & -5.7 & TYRA:299 & $\begin{array}{c}\text { TRPA:441, HISA:600, } \\
\text { PHEA:575, TRPA:406, } \\
\text { PHEA:450 }\end{array}$ \\
\hline 3 & Rheagenine & -8.3 & ASPA:203 & $\begin{array}{c}\text { LYSA:480, ASPA:542. } \\
\text { TRPA:406 }\end{array}$ \\
\hline 4 & Alpha humulene & -6 & No Interactions & PHEA:575, ALAA:576 \\
\hline 5 & Isoaromadendrene epoxide & -5.8 & GLUA:333 & ARGA:334 \\
\hline 6 & $\begin{array}{l}\text { Butanedioic acid diethyl } \\
\text { ester }\end{array}$ & -5.9 & $\begin{array}{l}\text { THRA:205, } \\
\text { ASPA:203, } \\
\text { THRA:204 } \\
\end{array}$ & $\begin{array}{l}\text { ILEA:328, TRPA:406, } \\
\text { TYRA:605, PHEA:575, } \\
\text { ALAA:576, TYRA:299 }\end{array}$ \\
\hline 7 & $\begin{array}{l}9,12,15 \text {-octadecatrienoic } \\
\text { acid }\end{array}$ & -5.5 & HISA:600 & $\begin{array}{c}\text { TYRA:299, PHEA:575, } \\
\text { ALAA:576, TYRA:605, } \\
\text { ASPA:443 } \\
\end{array}$ \\
\hline 8 & Beta-ionone & -5.3 & GLNA:603 & TRPA:406 \\
\hline 9 & 9,12 -octadecadienoic acid & -5.4 & THRA:205 & $\begin{array}{c}\text { LYSA:480, TRPA:406, } \\
\text { PHEA:575, PHEA:450, } \\
\text { ILEA:328, TYRA:299 }\end{array}$ \\
\hline 10 & Alpha muurolol & -5.4 & ASPA:203 & PHEA:450, LYSA:480 \\
\hline 11 & Bauhinione & -6.4 & $\begin{array}{l}\text { LYSA:480, } \\
\text { PHEA:450, } \\
\text { GLUA:404 }\end{array}$ & No Interactions \\
\hline 12 & Beta-sitosterol & -7.5 & GLUA:404 & PHEA:575, PHEA:450 \\
\hline 13 & Kaempferol-3-glucoside & -7.1 & $\begin{array}{l}\text { ARGA:526, } \\
\text { ASPA:542, } \\
\text { ASPA: } 443 \\
\end{array}$ & $\begin{array}{c}\text { TYRA:299, PHEA:575, } \\
\text { TRPA:406 }\end{array}$ \\
\hline 14 & $\begin{array}{l}\text { ACARBOSE } \\
\text { (STANDARD) }\end{array}$ & -7.1 & No Interactions & $\begin{array}{l}\text { TYRA:301, GLUA:333, } \\
\text { ASPA:340, GLUA:300, } \\
\text { ASPA:329, GLYA:302 }\end{array}$ \\
\hline
\end{tabular}

All the plant components made hydrogen bond interactions with aminoacids PHEA:10, ALAA:63, ARGA:18, PROA:61, SERA:66, ARGA:18, VALA:64, VALA:87, ALAA:88, ARGA:11, PROA:275, VALA:81, TYRA:62, ASNA:67, SERA:91, PROA:84 in the active site of 1T02 target. Binding energies of all the compounds were found between -6.32 to $-8.7 \mathrm{kcal} / \mathrm{mol}$ were illustrated in (Table 8). Among all compounds, Rheagenine, Beta-sitosterol, Bauhinione, Quercetin showed more binding energies than the Standard Simvastatin $(-7.7 \mathrm{kcal} / \mathrm{mol})$.

As a result of the docking process on 6 different targets, the selected plant components like Quercetin, Beta-sitosterol, and Rheagenine were observed to show better binding energy values against all the 5 targets except 6VYO as shown in (Table 9).

This present study has paved the way in understanding that some of the plant components of Bauhinia acuminata may act as potential inhibitors against enzyme targets namely 2ITY, 1A52, 3L4U, IT02, 5-COX except 6VYO. 
As a result of the docking process on 6 different targets, the selected plant components like Quercetin, Beta-sitosterol, and RHEAGENINE were observed to show good binding energy values against all the 5 targets except 6VYO. This research can further provide better insights in identifying and designing potential lead candidates against Lung cancer, breast cancer, diabetes, obesity, and inflammation in the area of drug discovery.

Table 6. Calculated binding energy values and interaction properties of the plant constituents of Bauhinia acuminata the target $1 \mathrm{~T} 02$.

\begin{tabular}{|c|c|c|c|c|}
\hline S.No & Plant Constituent & $\begin{array}{c}\text { Binding } \\
\text { energy } \\
\text { (kcal/mol) }\end{array}$ & $\begin{array}{l}\text { Hydrogen Bond } \\
\text { Interactions }\end{array}$ & Alkyl/ $\pi$ Alkyl Interactions \\
\hline 1 & Quercetin & -7.8 & $\begin{array}{l}\text { ASNA:67, SERA:91, } \\
\text { ALAA: } 88\end{array}$ & VALA:87, ARGA:11 \\
\hline 2 & Neophytadiene & -6.3 & No Interactions & $\begin{array}{c}\text { VALA:64, ALAA:63, } \\
\text { ARGA:18, LEUA:19, ILEA:22, } \\
\text { LEUA:36, PHEA:10, PROA:15 }\end{array}$ \\
\hline 3 & Rheagenine & -8.7 & $\begin{array}{l}\text { SERA:66, ALAA:88, } \\
\text { ARGA: } 18\end{array}$ & $\begin{array}{c}\text { VALA:87, ALAA:63, PHEA:10, } \\
\text { ARGA:11 }\end{array}$ \\
\hline 4 & Alpha humulene & -7.1 & LEUA:19, ARGA:18 & No Interactions \\
\hline 5 & $\begin{array}{l}\text { Isoaromadendrene } \\
\text { epoxide }\end{array}$ & -7.3 & PROA:275, VALA:81 & VALA:278, TRPA:284 \\
\hline 6 & $\begin{array}{l}\text { Butanedioic acid } \\
\text { diethyl ester }\end{array}$ & -6.6 & ARGA:18, VALA:64 & $\begin{array}{c}\text { VALA:87, ALAA:88, } \\
\text { ARGA:11, LEUA:19, ILEA:22, } \\
\text { ILEA:344, TYRA:62, LEUA:36, } \\
\text { LEUA:19 }\end{array}$ \\
\hline 7 & $\begin{array}{l}9,12,15- \\
\text { octadecatrienoic } \\
\text { acid }\end{array}$ & -6.7 & $\begin{array}{l}\text { SERA:66, SERA:91, } \\
\text { VALA:64 }\end{array}$ & $\begin{array}{c}\text { VALA:87, PHEA:10, ALAA:63, } \\
\text { PROA:15, ARGA:18, LEUA:19, } \\
\text { ILEA:22, LEUA:36, TYRA:62 }\end{array}$ \\
\hline 8 & Beta-ionone & -6.3 & ARGA: 18 & ILEA:22, VALA:64, LEUA:19 \\
\hline 9 & $\begin{array}{c}9,12- \\
\text { octadecadienoic } \\
\text { acid } \\
\end{array}$ & -6.4 & $\begin{array}{c}\text { SERA:66, SERA:91, } \\
\text { VALA:87, ALAA:88, } \\
\text { ARGA: } 11\end{array}$ & $\begin{array}{c}\text { VALA:64, ALAA:63, } \\
\text { ARGA:18, LEUA:19, ILEA:22, } \\
\text { LEUA:36, PHEA:10 }\end{array}$ \\
\hline 10 & Alpha muurolol & -7.6 & TYRA:62 & $\begin{array}{c}\text { ILEA:344, VALA:64, LEUA:36, } \\
\text { ILEA:22, LEUA:19, PROA:15, } \\
\text { ARGA:18 }\end{array}$ \\
\hline 11 & Bauhinione & -8 & $\begin{array}{l}\text { ASNA:67, SERA:66, } \\
\text { SERA:91, ARGA:11 }\end{array}$ & ALAA:88 \\
\hline 12 & Beta-sitosterol & -8.3 & PROA:84 & $\begin{array}{c}\text { PROA:61, ALAA:63, VALA:87, } \\
\text { ARGA:11 }\end{array}$ \\
\hline 13 & $\begin{array}{l}\text { Kaempferol-3- } \\
\text { glucoside }\end{array}$ & -7.6 & No Interactions & LEUA:19, ILEA:22, VALA:87 \\
\hline 14 & $\begin{array}{l}\text { SIMVASTATIN } \\
\text { (STANDARD) }\end{array}$ & -7.7 & $\begin{array}{l}\text { PHEA:10, ALAA:63, } \\
\text { ARGA:18, PROA:61 }\end{array}$ & VALA:87 \\
\hline
\end{tabular}


Table 7. Calculated binding energy values and interaction properties of the plant constituents of Bauhinia acuminata the target 5COX

\begin{tabular}{|c|c|c|c|c|}
\hline S.No & Plant Constituent & $\begin{array}{l}\text { Binding energy } \\
(\mathrm{kcal} / \mathrm{mol})\end{array}$ & $\begin{array}{l}\text { Hydrogen Bond } \\
\text { Interactions }\end{array}$ & $\begin{array}{l}\text { Alkyl/ } \pi \text { Alkyl } \\
\text { Interactions }\end{array}$ \\
\hline 1 & Quercetin & -8.4 & $\begin{array}{l}\text { TYRB:373, } \\
\text { GLNB:374, } \\
\text { ARGA:376, } \\
\text { ASNA:375, } \\
\text { TYRA:373 }\end{array}$ & PHEA:142 \\
\hline 2 & Neophytadiene & -5 & LEUA:145 & $\begin{array}{c}\text { PHEB:142, LEUB:145, } \\
\text { PHEA:142 }\end{array}$ \\
\hline 3 & Rheagenine & -9.9 & $\begin{array}{l}\text { ASNB:375, } \\
\text { ASNA:375, } \\
\text { ARGB:376, } \\
\text { LEUB: } 145, \\
\text { LEUA: } 145\end{array}$ & No Interactions \\
\hline 4 & Alpha humulene & -6.5 & PHEB:142 & No Interactions \\
\hline 5 & $\begin{array}{l}\text { Isoaromadendrene } \\
\text { epoxide }\end{array}$ & -7.1 & $\begin{array}{l}\text { PHEB:142, } \\
\text { ARGB:376, } \\
\text { LEUA:145 } \\
\end{array}$ & No Interactions \\
\hline 6 & $\begin{array}{c}\text { Butanedioic acid diethyl } \\
\text { ester }\end{array}$ & -6.2 & PROB:538 & PHEA:142 \\
\hline 7 & $\begin{array}{c}9,12,15 \text {-octadecatrienoic } \\
\text { acid }\end{array}$ & -5.2 & HISB:226 & LEUA:145, PHEA:142 \\
\hline 8 & Beta-ionone & -6.2 & $\begin{array}{l}\text { GLNA: } 241, \\
\text { TRPB:139, } \\
\text { LYSA:333, } \\
\text { GLUB: } 140\end{array}$ & LEUA:238 \\
\hline 9 & 9,12-octadecadienoic acid & -5.4 & $\begin{array}{l}\text { GLYA:227, } \\
\text { ASNA:375 }\end{array}$ & $\begin{array}{c}\text { LEUA:145, PHEB:142, } \\
\text { LEUB:145 } \\
\end{array}$ \\
\hline 10 & Alpha muurolol & -6.7 & $\begin{array}{l}\text { GLYB:225, } \\
\text { ASNB:375 }\end{array}$ & $\begin{array}{c}\text { HISB:226, LEUB:145, } \\
\text { PHEA:142 } \\
\end{array}$ \\
\hline 11 & Bauhinione & -8.1 & $\begin{array}{l}\text { ASNA:375, } \\
\text { LEUA: } 145, \\
\text { GLYA: } 225\end{array}$ & PROA:538, PHEB:142 \\
\hline 12 & Beta-sitosterol & -9.3 & GLYA:536 & PHEB:142 \\
\hline 13 & Kaempferol-3-glucoside & -8.1 & $\begin{array}{l}\text { ASNB:375, } \\
\text { VALB:228 }\end{array}$ & PROB:538 \\
\hline 14 & ASPRIN (STANDARD) & -5.8 & $\begin{array}{l}\text { LYSA:333, } \\
\text { GLNA:241, } \\
\text { GLUB:140, } \\
\text { ASNB: } 144\end{array}$ & No Interactions \\
\hline
\end{tabular}

Table 8. Calculated binding energy values and interaction properties of the plant constituents of Bauhinia acuminata the target $6 \mathrm{VYO}$

\begin{tabular}{|c|c|c|c|c|}
\hline S.No & Plant Constituent & $\begin{array}{c}\text { Binding } \\
\text { energy } \\
\text { (kcal/mol) }\end{array}$ & $\begin{array}{l}\text { Hydrogen Bond } \\
\text { Interactions }\end{array}$ & Alkyl/ $\pi$ Alkyl Interactions \\
\hline 1 & Quercetin & -8.1 & ASNB:153 & ARGA:107, TRPB:52, ALAA:55 \\
\hline 2 & Neophytadiene & -5.7 & No Interactions & $\begin{array}{c}\text { ILEB:74, ILEB:157, VALA:158, } \\
\text { TRPB:52, CLA:202, ILEB:146 }\end{array}$ \\
\hline 3 & Rheagenine & -7.9 & THRB:76, ASNB:77 & ILEB:157, VALA:158 \\
\hline 4 & Alpha humulene & -8.7 & No Interactions & No Interactions \\
\hline 5 & $\begin{array}{l}\text { Isoaromadendrene } \\
\text { epoxide }\end{array}$ & -9.7 & $\begin{array}{l}\text { VALA:158, ILEB:157, } \\
\text { ILEB:146 }\end{array}$ & TRPB:52 \\
\hline
\end{tabular}


Table 8 (continued). Calculated binding energy values and interaction properties of the plant constituents of Bauhinia acuminata the target 6VYO.

\begin{tabular}{|c|c|c|c|c|}
\hline S.No & Plant Constituent & $\begin{array}{c}\text { Binding } \\
\text { energy } \\
(\mathbf{k c a l} / \mathbf{m o l})\end{array}$ & $\begin{array}{l}\text { Hydrogen Bond } \\
\text { Interactions }\end{array}$ & Alkyl/ $\pi$ Alkyl Interactions \\
\hline 6 & $\begin{array}{l}\text { Butanedioic acid } \\
\text { diethyl ester }\end{array}$ & -6.7 & $\begin{array}{c}\text { ALAA:55, ARGA:107, } \\
\text { ARGA:149, ASNB:154, } \\
\text { ASNB:153 }\end{array}$ & $\begin{array}{c}\text { ILEB:157, ALAA:50, TYRA:109, } \\
\text { VALA:158, TRPB:52 }\end{array}$ \\
\hline 7 & $\begin{array}{c}9,12,15- \\
\text { octadecatrienoic acid }\end{array}$ & -6.4 & $\begin{array}{c}\text { ARGA:92, ARGA:107, } \\
\text { THRB: } 148\end{array}$ & CLA:202, TRPB:52, ILEB:146 \\
\hline 8 & Beta-ionone & -7.8 & ALAA:55,ARGA:107 & TRPB:52 \\
\hline 9 & $\begin{array}{l}\text { 9,12-octadecadienoic } \\
\text { acid }\end{array}$ & -6.3 & $\begin{array}{c}\text { ALAB:155, ALAA:156, } \\
\text { ASNB:154 }\end{array}$ & $\begin{array}{l}\text { ILEB:146, ILEB:157, CLA:202, } \\
\text { ALAA:55, TYRA:109, TRPB:52 }\end{array}$ \\
\hline 10 & Alpha muurolol & -7.8 & No Interactions & $\begin{array}{c}\text { ILEB:157, VALA:158, TRPB:52, } \\
\text { ALAA:55 }\end{array}$ \\
\hline 11 & Bauhinione & -6.9 & $\begin{array}{c}\text { SERA:105, SERB:79, } \\
\text { THRB:141, HISA:59, } \\
\text { ASPB:81 }\end{array}$ & $\begin{array}{c}\text { HISB:145, ASPB:144, LYSA:102, } \\
\text { PROB:142 }\end{array}$ \\
\hline 12 & Beta-sitosterol & -7.2 & No Interactions & TYRA:172, PROB:80, PROB:162 \\
\hline 13 & $\begin{array}{l}\text { Kaempferol-3- } \\
\text { glucoside }\end{array}$ & -7.6 & ALAA:55, ASNB:75 & ILEB:146, TRPB:52, ARGA:107 \\
\hline 14 & $\begin{array}{l}\text { N3 INHIBITOR of } \\
\text { 6LU7 (STANDARD) }\end{array}$ & -8.7 & $\begin{array}{c}\text { ALAA:50, TYRA:111, } \\
\text { ARGA:88, TYRA:109, } \\
\text { ALAA:55, ARGA:107, } \\
\text { ASNB:153 }\end{array}$ & PROA:117, TRPB:52 \\
\hline
\end{tabular}

Table 9. Binding energy values of prioritized plant constituents of Bauhinia acuminata againsts elected targets

\begin{tabular}{|c|c|c|c|c|c|c|}
\hline Target protein & 2ITY & $1 \mathrm{A52}$ & 3L4U & IT02 & $5 \mathrm{COX}$ & 6VYO \\
\hline Plant constituent & \multicolumn{6}{|c|}{ Binding energy $(\mathrm{kcal} / \mathrm{mol})$} \\
\hline Quercetin & -8 & -8.5 & -7.3 & -7.8 & -8.4 & -8.1 \\
\hline Beta sitosterol & -8.4 & -7 & -7.5 & -8.3 & -9.3 & -7.2 \\
\hline Rheagenine & -9.2 & -7.2 & -8.3 & -8.7 & -9.9 & -7.9 \\
\hline Standard & -7.1 & -6.2 & -7.1 & -7.7 & -5.8 & -8.7 \\
\hline
\end{tabular}

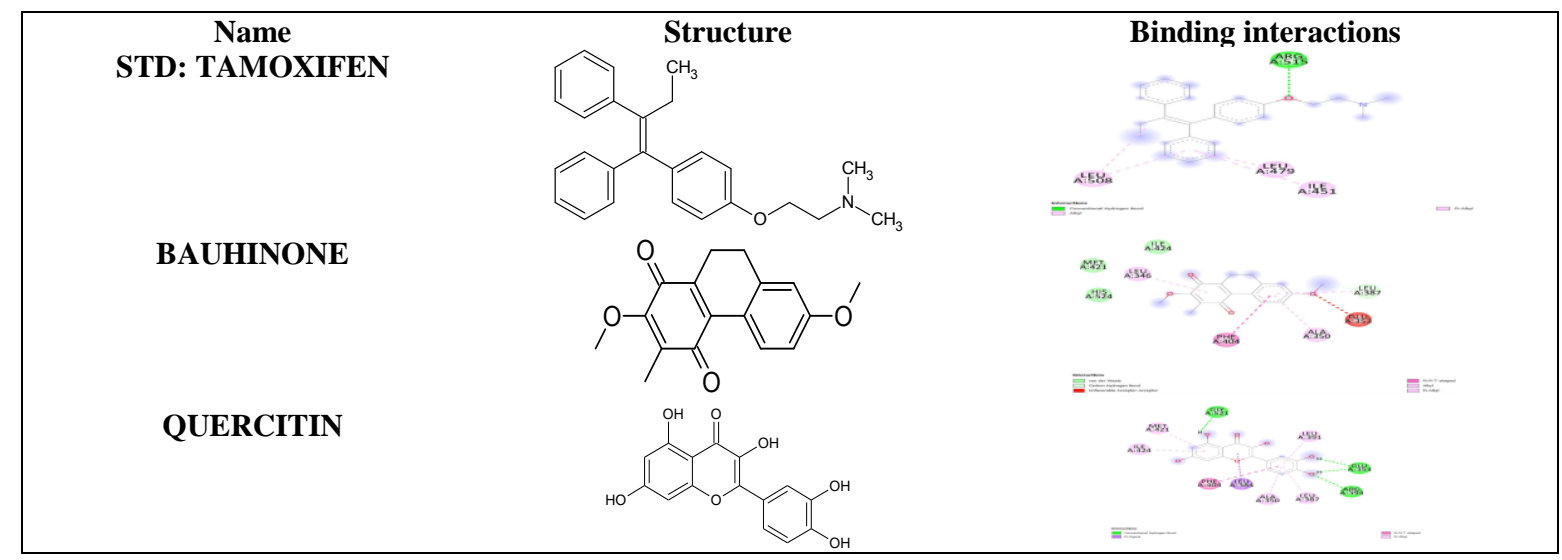

Figure 1. Docking interaction of Tamoxifen (Standard), Bauhinione and Quercetin within the active site of target 1A52 


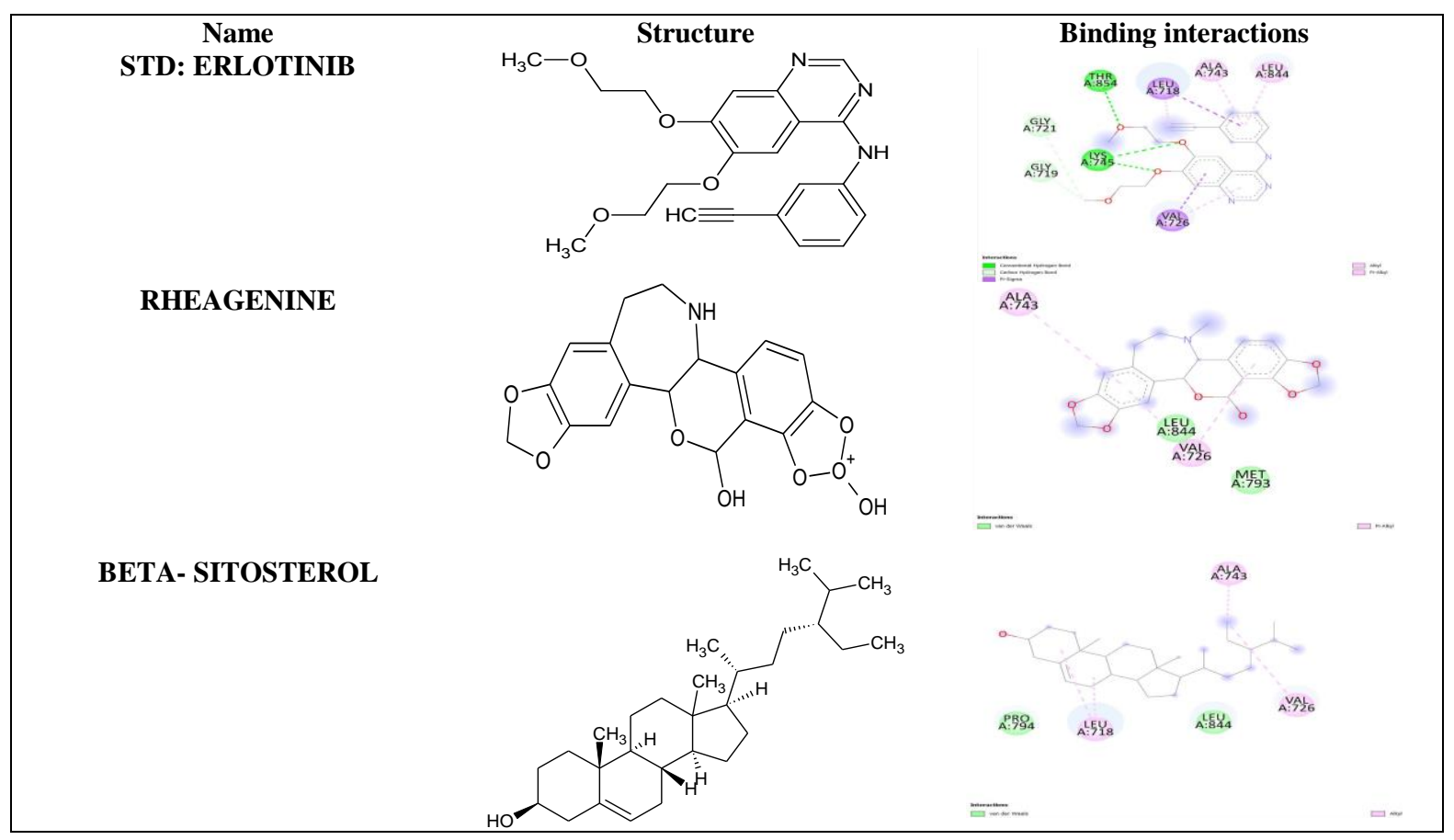

Figure 2. Docking interaction of Erlotinib (Standard), RHEAGENINE and Beta-sitosterol within the active site of target 2ITY

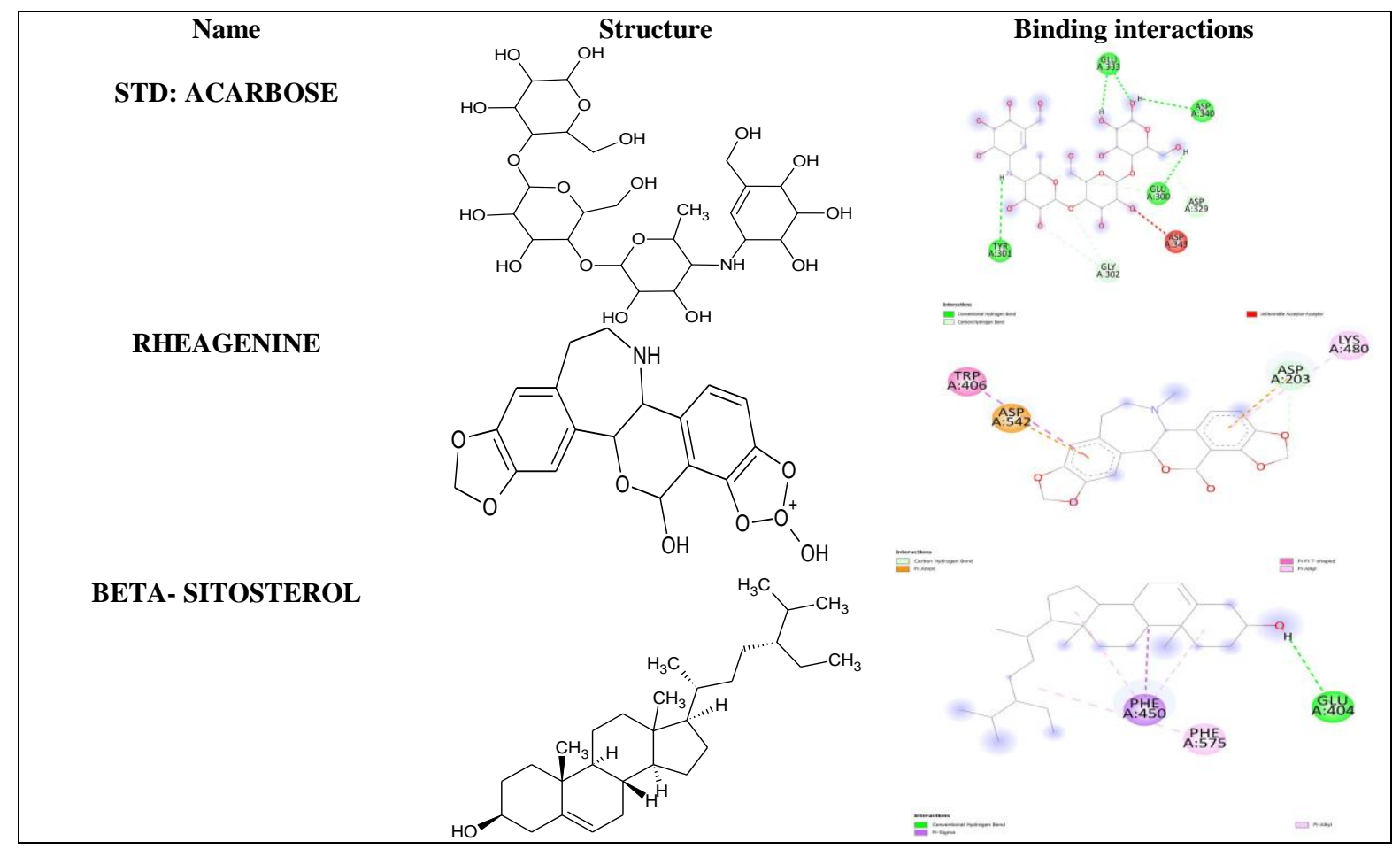

Figure 3. Docking interaction of Acarbose (Standard), RHEAGENINE and Beta-sitosterol within the active site of target $3 \mathrm{~L} 4 \mathrm{U}$ 


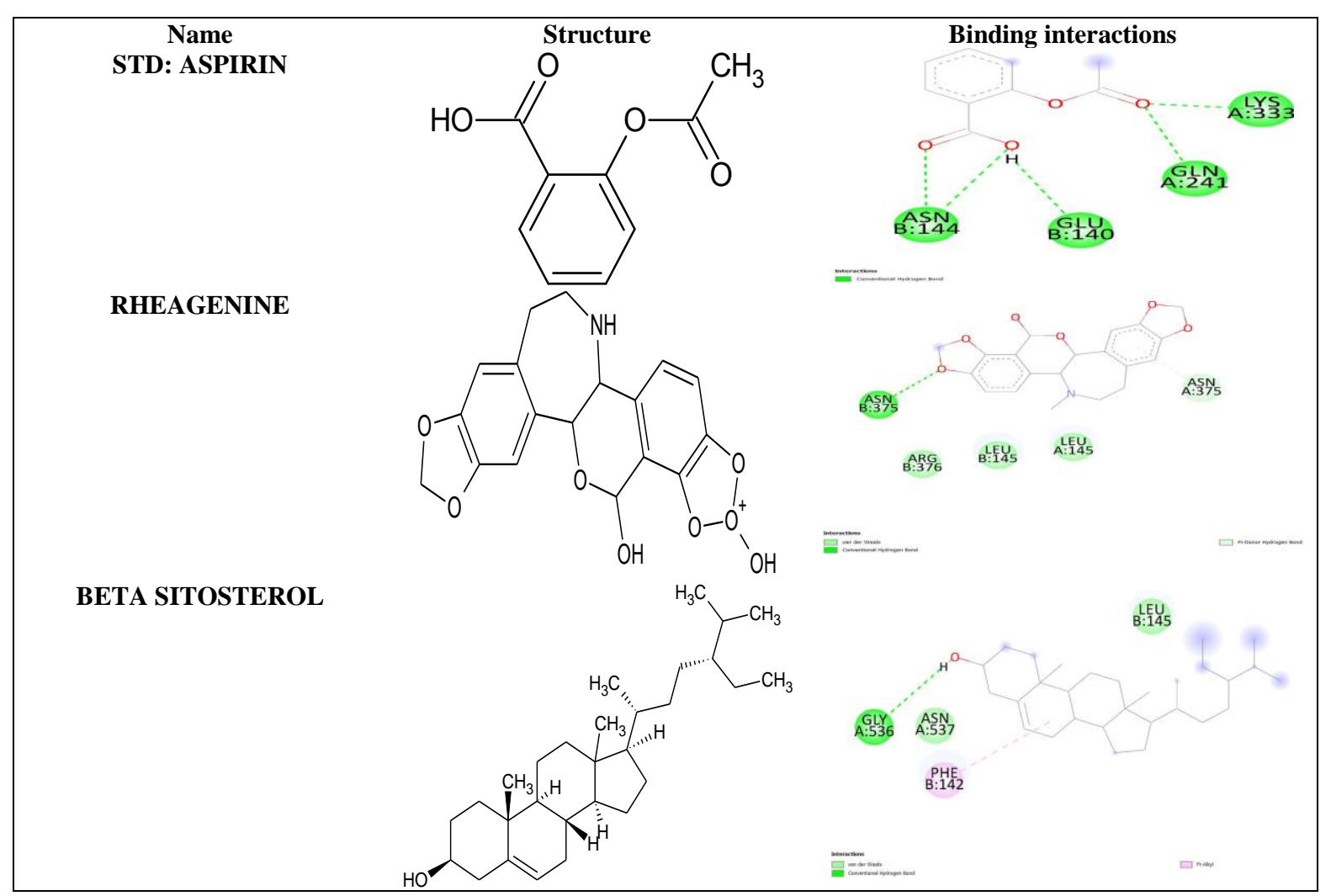

Figure 4. Docking interaction of Aspirin (Standard), RHEAGENINE and Beta-sitosterol within the active site of target 5COX

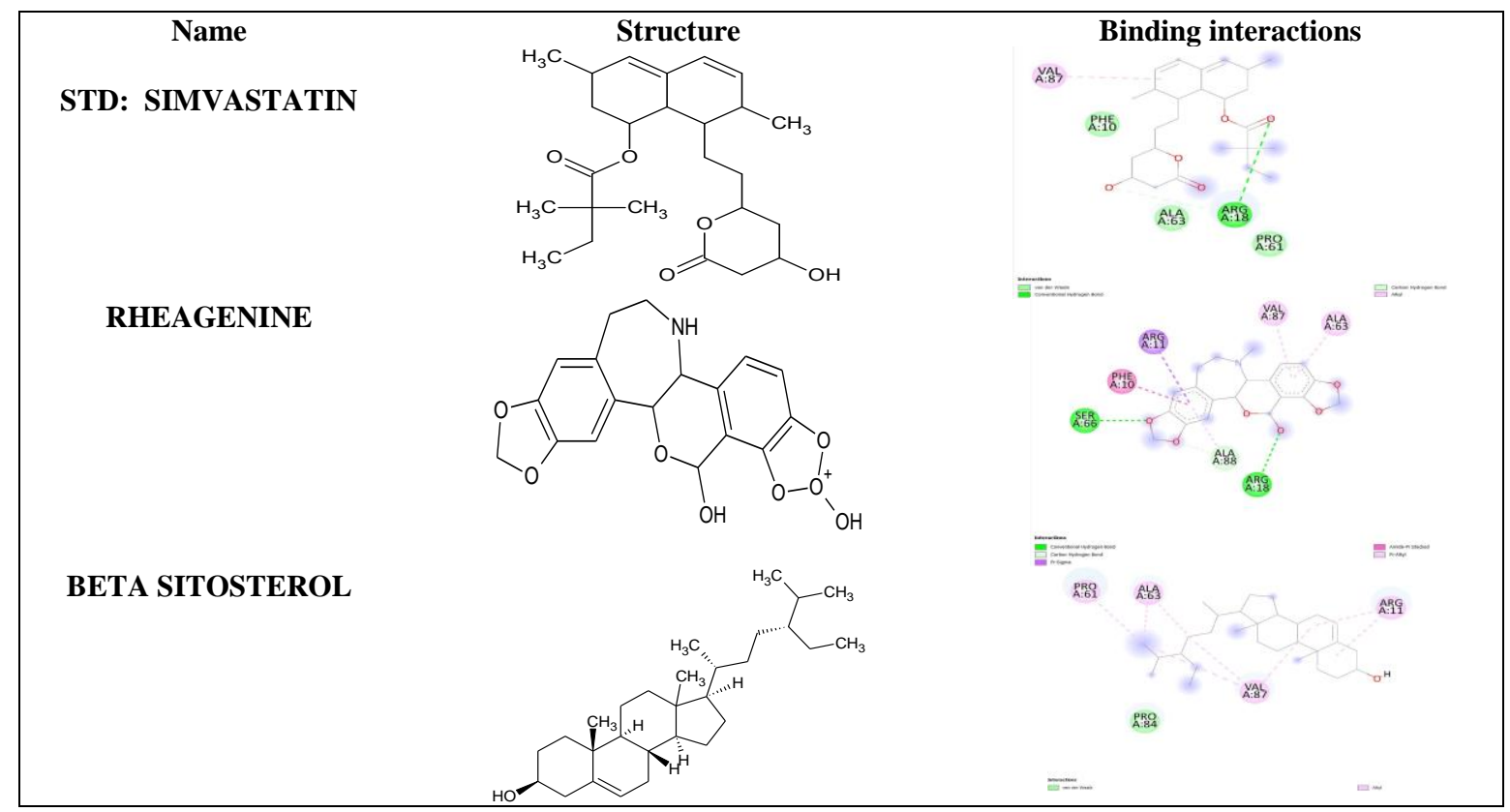

Figure 5. Docking interaction of Simvastatin (Standard), RHEAGENINE and Beta-sitosterol within the active site of target $1 \mathrm{~T} 02$ 


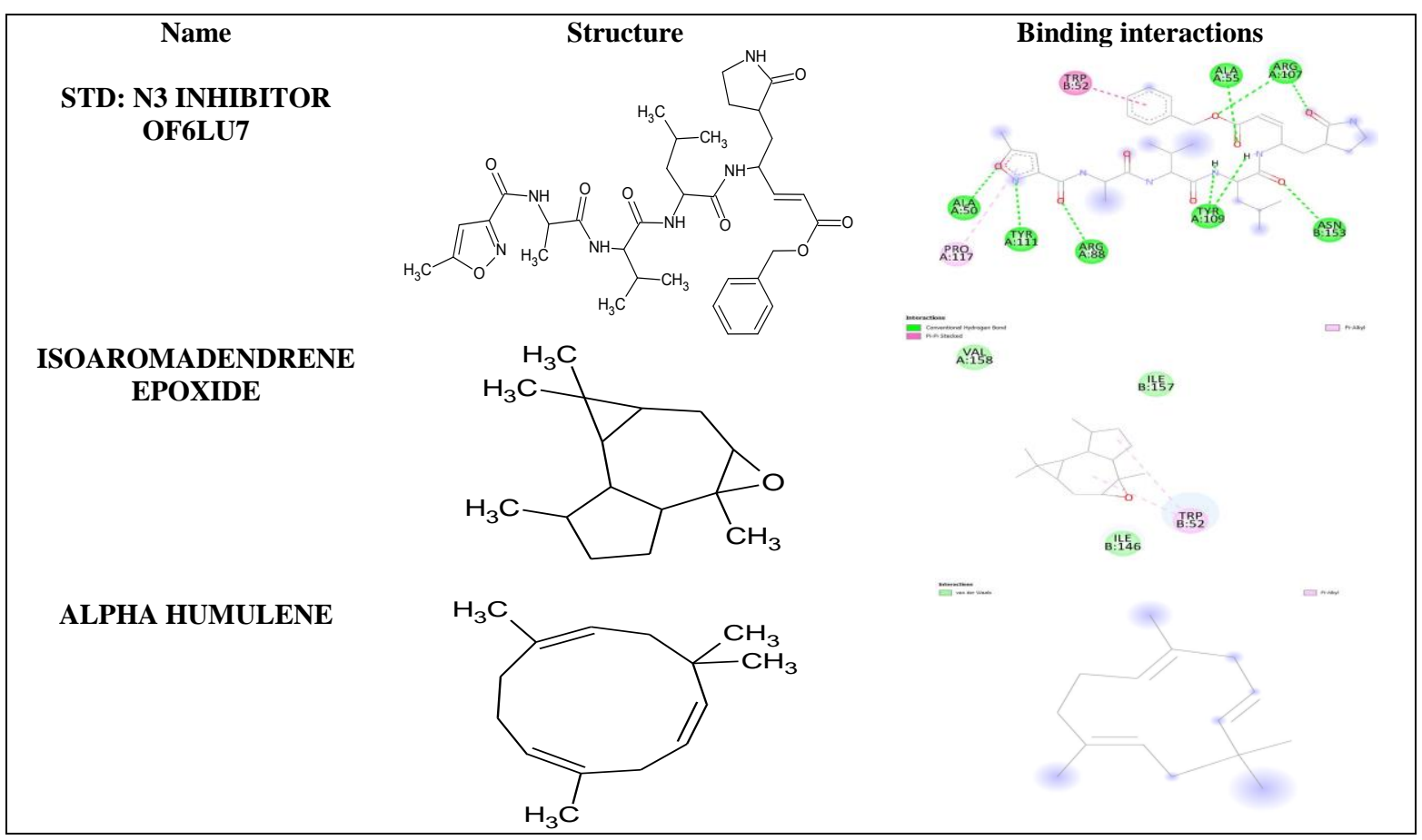

Figure 6. Docking interaction of N3 Inhibitor of 6LU7(Standard), Isoaromadendrene epoxide and Alpha Humulene within the active site of target 6VYO

\section{ACKNOWLEDGEMENT}

The authors are thankful to the Principal and Management of Sarojini Naidu Vanita Pharmacy Maha Vidyalaya, Osmania University, Hyderabad, India, for providing research facilities.

\section{AUTHOR CONTRIBUTIONS}

Concept: N.K.; Design: V.K., M.S.; Supervision: N.K.; Resources: V.K., M.S.; Materials: M.S., V.K.; Data Collection and/or processing: N.K., V.K., M.S.; Analysis and/or interpretation: N.K., M.S.; Literaturereview: N.K.; Manuscript writing: N.K., V.K., M.S.; Critical review: N.K., V.K, M.S.

\section{CONFLICT OF INTEREST}

The authors declare no conflict of interest.

\section{ETHICS COMMITTEE APPROVAL}

The authors declare that the ethics committee approval is not required for this study. 


\section{REFERENCES}

1. Qi, Z. (2013). WHO traditional medicine strategy: 2014-2023. Geneva: World HealthOrganization.

2. Chintamunnee, V., Mahomoodally, M.F. (2012). Herbal medicine is commonly used against noncommunicable diseases in the tropical island of Mauritius. Journal of Herbal Medicine, 2(4), 113125. [CrossRef]

3. Gurib-Fakim, A. (2006). Medicinal plants: traditions of yesterday and drugs of tomorrow. Molecular Aspects of Medicine, 27(1), 1-93. [CrossRef]

4. Lin, L.L., Shan, J.J., Xie, T., Xu, J.Y., Shen, C.S., Di, L.Q., Chen, J.B., Wang, S.C. (2016). Application of traditional Chinese medical herbs in prevention and treatment of the respiratory syncytial virus. Evidence Based Complementary Alternative Medicine, 2016, 6082729. [CrossRef]

5. $\quad$ Lin T.Z., Hsu W.C., Lin C.C (2014). Antiviral natural products and herbal medicines. Journal of Traditional and Complement Medicine, 4(1), 24-35. [CrossRef]

6. Sebastian, D., Nirmal, S. (2020). Pharmacognostic standardization and preliminary phytochemical studies of Bauhinia acuminata. Journal of Pharmacognosy and Phytochemistry, 9(2), 2150-2154.

7. Sebastian, D., Fleming, A.T. (2017). Synthesis of silver nanoparticles from bauhinia acuminata aqueous leaf extract and molecular docking analysis of various cancer receptors. International Journal of Science and Research, 6(3), 50-55.

8. Roy, M.N., Naz, T., Khan, A., Ali, H. (2017). Antidiabetic potential of methanolic extract of leave and bark of bangladeshi medicinal plant Bauhinia acuminata L on mice. Journal of Diabetes and Metabolism, 8(9), 762. [CrossRef]

9. Govindula, A., Reddy, M.S., Manjula, M., Reddy, M.S., Kalyani, P. (2019). Invivo antihyperlipidemic activity and preliminary phytochemical screening of Bauhinia acuminata. International Journal of Pharma Sciences and Scientific Research, 11(2), 1000-1008.

10. Dutta, S., Hossain, S., Islam, E., Haque, U., Parvin, S. (2020). Assessment of antioxidant and anti-inflammatory activities of stem bark of Bauhinia acuminata L. Bio medical Journal of Scientific and Technical Research, 24(5), 18519-18527. [CrossRef]

11. Reddy, P.S., Lokhande, K.B., Nagar, S., Reddy, V.D., Murthy, P.S., Swamy, K.V. (2018). Molecularmodeling, docking, dynamicsandsimulation of gefitinibanditsderivativeswith EGFR in non-smallcelllungcancer. Current Computer Aided Drug Design, 14(3), 246-252. [CrossRef]

12. Tecalco-Cruz, A.C., Ramírez-Jarquín, J.O., Cruz-Ramos, E. (2019). Estrogen receptor alpha and its ubiquitination in breast cancer cells. Current Drug Targets, 20(6), 690-704.

13. Dirir, A.M., Daou, M., Yousef, A.F., Yousef, L.F. (2021). A review of alpha-glucosidase inhibitors from plants as potential candidates for the treatment of type-2 diabetes. Phytochemistry Reviews. [CrossRef] 
14. März, W., Köenig, W. (2003). HMG CoA Reductase inhibition: anti-inflammatory effects beyond lipid lowering? Journal of Cardiovascular Risk, 10(3), 169-179. [CrossRef]

15. Kurumbail, R.G., Stevens, A.M., Gierse, J.K., McDonald, J.J., Stegeman, R.A., Pak, J.Y., Gildehaus, D., Miyashiro, J.M., Penning, T.D., Seibert, K., Isakson, P.C., Stallings, W.C. (1996). Structural basis for selective inhibition of cyclooxygenase-2 by anti-inflammatory agents. Nature, 384(6610), 644-648. [CrossRef]

16. Ercan, S., Ercan, Ç. (2021). A molecular docking study of potential inhibitors and repurposed drugs against SARS-CoV-2 main protease enzyme. Journal of the Indian Chemical Society, 98(3), 100041. [CrossRef]

17. Protein Data Bank (PDB) [CrossRef]

18. AutoDockVina. v.1.2.0, the free GUI for AutoDock Vina. [CrossRef]

19. Pharmit: interactive exploration of chemical space. [CrossRef]

20. MuniSireesha, S., Bhowmik, D., Soujanya, D., Brijitha, G., AndJyothi, V. (2021). Computational validation of tacrine analogs as antialzheimer's agents against acetylcholinesterases. International Journal of Biology, Pharmacy and Allied Sciences, 10(10), 243-254. [CrossRef]

21. Barnum, D., Greene, J., Smellie, A., Sprague, P. (1996). Identification of common functional configurations among molecules. Journal of Chemical Information and Computer Sciences, 36(3), 563-571. [CrossRef]

22. Kudumula, Divya, N., Sravika, N., Priya, S., Anusha, P., Jyotsna, M.S. (2021). Molecular properties, biological activity results, and toxicity predictions of the plant components present in Bauhinia Acuminata. International Journal of Scientific Research and Management, 9(7), 408414. [CrossRef] 